\title{
Phenomenological implications of the intrinsic charm in the $Z$ boson production at the $\mathrm{LHC}$
}

\author{
G. Bailas ${ }^{1,3}$, V. P. Gonçalves ${ }^{2,3, a}$ \\ ${ }^{1}$ Laboratoire de Physique Corpusculaire, Université Blaise Pascal, CNRS/IN2P3, 63177 Aubière Cedex, France \\ ${ }^{2}$ Department of Astronomy and Theoretical Physics, Lund University, 22362 Lund, Sweden \\ ${ }^{3}$ High and Medium Energy Group, Instituto de Física e Matemática, Universidade Federal de Pelotas, Caixa Postal 354, Pelotas, RS 96010-900, \\ Brazil
}

Received: 18 December 2015 / Accepted: 5 February 2016 / Published online: 26 February 2016

(C) The Author(s) 2016. This article is published with open access at Springerlink.com

\begin{abstract}
In this paper we study the $Z, Z+$ jet, $Z+c$, and $Z+c+$ jet production in $p p$ collisions at the LHC considering different models for the intrinsic charm content of the proton. We analyze the impact of the intrinsic charm in the rapidity and transverse momentum distributions for these different processes. Our results indicated that differently from the other processes, the $Z+c$ cross section is strongly affected by the presence of the intrinsic charm. Moreover, we propose the analysis of the ratios $R(Z+c / Z) \equiv \sigma(Z+c) / \sigma(Z)$ and $R(Z+c / Z+$ jet $) \equiv \sigma(Z+c) / \sigma(Z+$ jet $)$ and we demonstrate that these observables can be used as a probe of the intrinsic charm.
\end{abstract}

A complete knowledge of the partonic structure of the hadrons is fundamental to make predictions for the Standard Model and to beyond Standard Model processes at hadron colliders. Since the early days of the parton model and of the first deep inelastic scattering (DIS) experiments, determining the precise form of the quark and gluon distributions of the nucleon has been a major goal of high energy hadron physics. Over the last 40 years huge progress has been achieved. In particular, data from HERA have dramatically improved our knowledge as regards the small- $x$ behavior of the parton distributions functions (PDFs) [1]. Another important improvement has occurred to our knowledge for the heavy quark contribution to the proton structure [2]. In the last years several groups have proposed different schemes to determine these distributions considering that the heavy quark component in PDFs can be perturbatively generated by gluon splitting (see e.g. [3]). This component is usually denoted the extrinsic heavy quark component. Moreover, the possibility of an intrinsic component has been studied in detail and included in the recent versions of the PDF parametrizations [4-7]. The hypothesis of intrinsic heavy quarks (IHQ) is a

a e-mails: barros@ufpel.edu.br; victor.goncalves@thep.lu.se natural consequence of the quantum fluctuations inherent to Quantum Chromodynamics (QCD) and amounts to assuming the existence of a $Q \bar{Q}(Q=c, b, t)$ as a non-perturbative component in the hadron wave function. A comprehensive review of the main characteristics of the IHQ models can be found in [8-10]. In the model proposed by Brodsky, Hoyer, Peterson and Sakay (BHPS) [11], the creation of the $Q \bar{Q}$ pair was studied in detail (for a discussion of other models for the IHQ see Refs. [12-15]). It was assumed that the nucleon light cone wave function has higher Fock states, the first one being $\mid q q q Q \bar{Q}>$. The probability of finding the nucleon in this configuration was given by the inverse of the squared invariant mass of the system. Because of the heavy quark mass, this probability as a function of the quark fractional momentum, $P(x)$, is very hard, as compared to the one obtained through the DGLAP evolution. Although this model predicts the $x$ dependence of the intrinsic components, its normalization should be constrained by fitting the experimental data and still is an open question. In particular, two recent global analysis of the PDFs [5,6] have analyzed the importance of an intrinsic heavy quark component to describe a wide range of hard scattering data and obtained distinct conclusions. Basically, the current situation is such that new and more precise data are necessary to probe the hypothesis of intrinsic heavy quarks [16].

The presence of an intrinsic heavy quark component is expected to directly modify the cross section of the processes which are initiated by heavy quarks and indirectly to other processes, since the presence of the IHQ modifies the contribution of the other partons due the momentum sum rule. One of the most striking properties of an IHQ state, such as $|u u d Q \bar{Q}\rangle$, is that the heavy constituents tend to carry the largest fraction of the momentum of the hadron. Consequently, in contrast to heavy quarks produced through usual perturbative QCD, which emerge with small longitudi- 

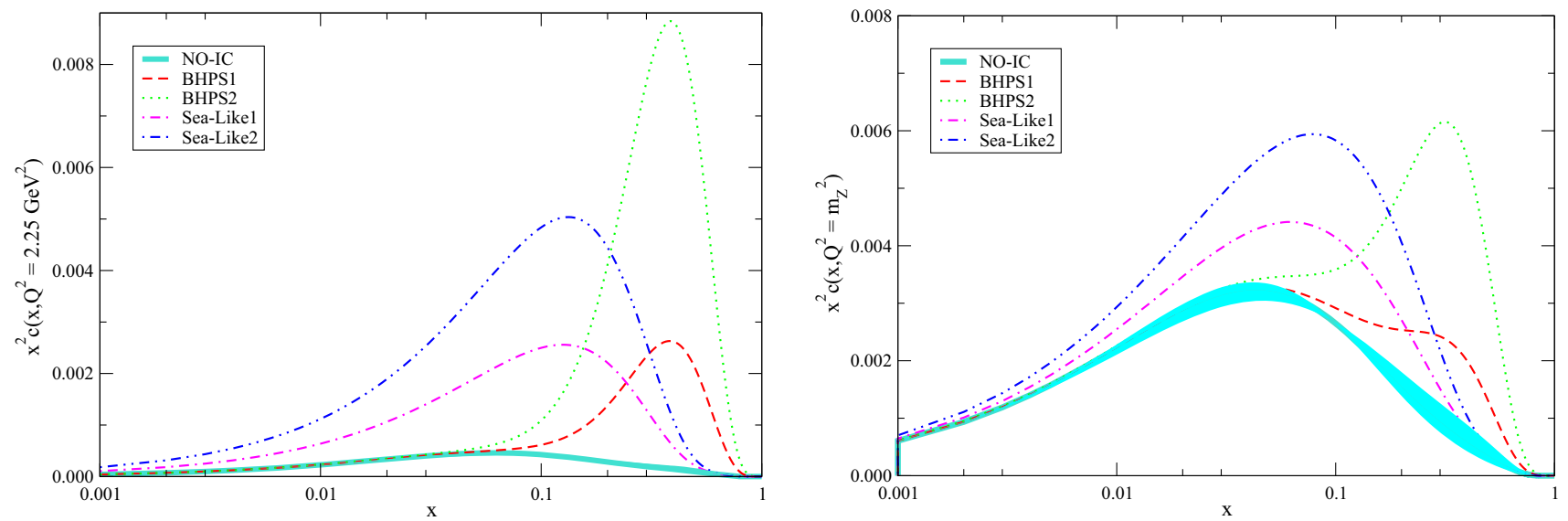

Fig. 1 Comparison between the different models for the intrinsic charm present in the CT14 parametrization for two different values of the hard scale $Q^{2}$
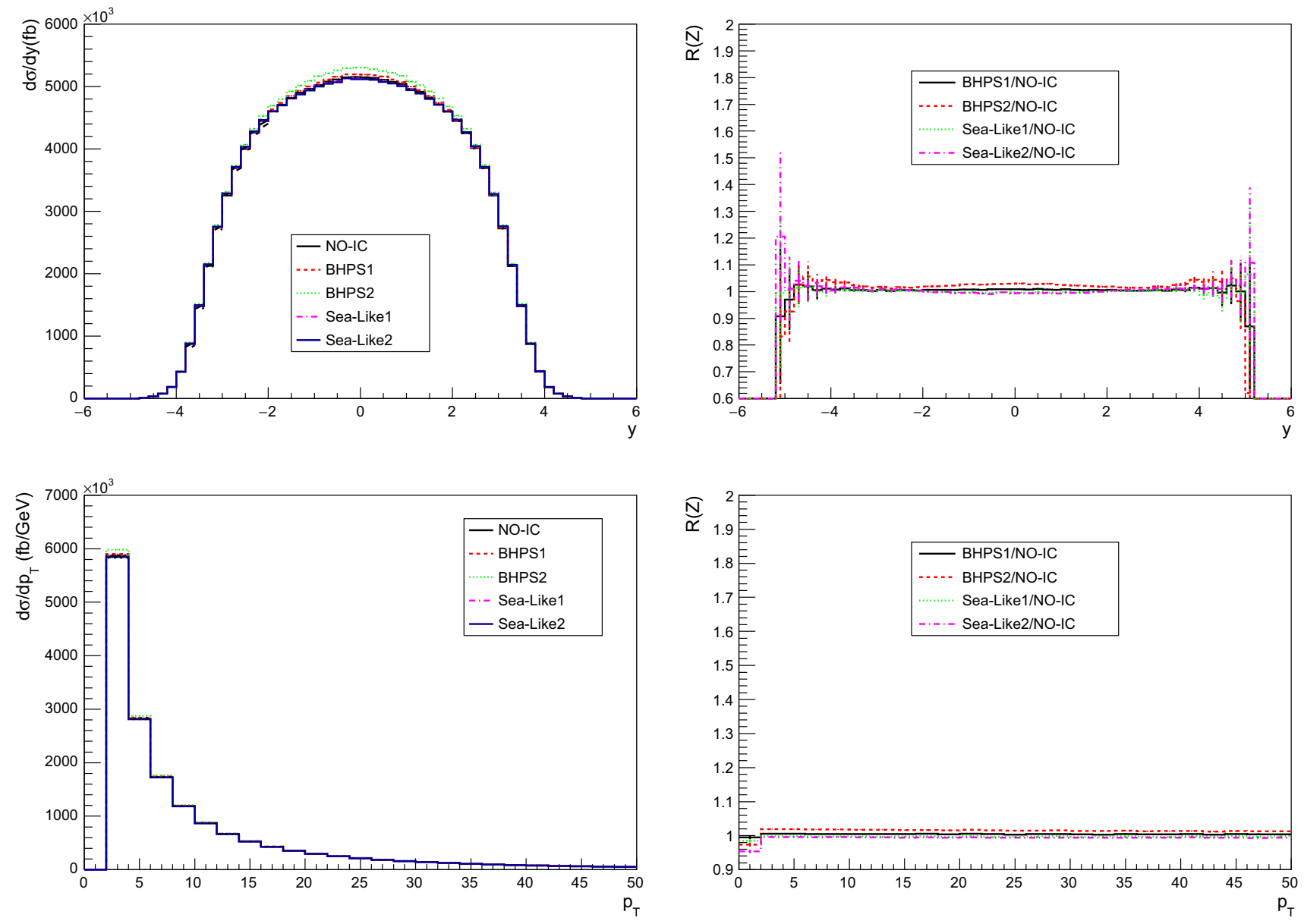

Fig. 2 Left panels Predictions for the rapidity and transverse momentum distributions for the $Z$ production in $p p$ collisions at $\sqrt{s}=7$ TeV. Right panels Rapidity and transverse momentum dependencies of the ratio between the IC and central NO-IC predictions

nal momentum, the intrinsic quark component gives rise to heavy mesons with large fractional momenta relative to the beam particles. Therefore, the existence of an intrinsic component is expected to modify, for instance, the $x_{F}$ and rapidity distribution of charmed particles (see, e.g. [17-19]). Moreover, it can also lead to Higgs production at high $x_{F}$ [20]. Many of these features have been discussed in the pioneering work on intrinsic heavy quark [11,21-23]. Another promis- 

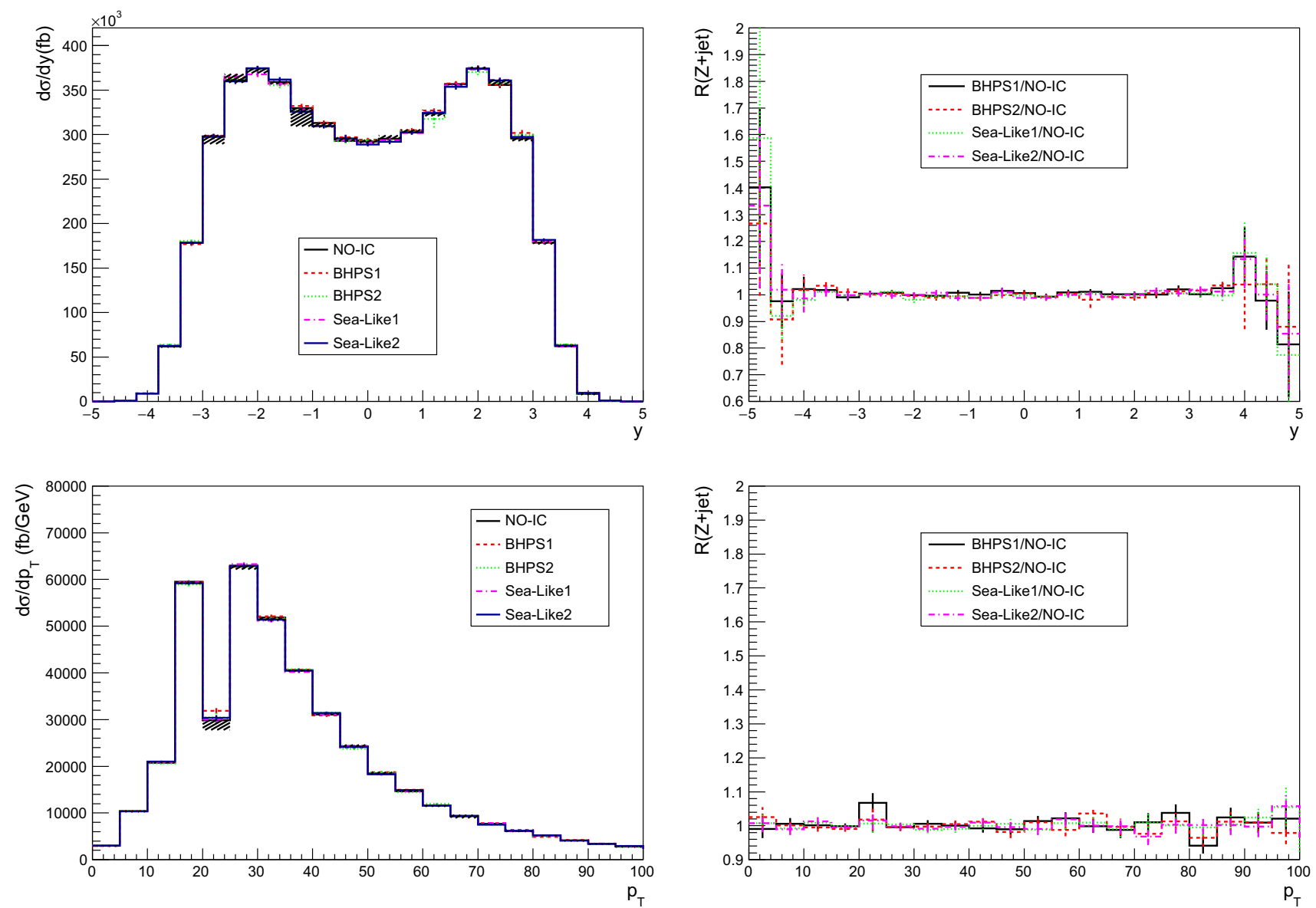

Fig. 3 Left panels Predictions for the rapidity and transverse momentum distributions for the $Z+$ jet production in $p p$ collisions at $\sqrt{s}=7$ TeV. Right panels Rapidity and transverse momentum dependencies of the ratio between the IC and central NO-IC predictions

ing observable to search the intrinsic heavy quark component is the associated production of a heavy quark with a gauge boson, which is strongly dependent on the heavy quark distribution. In recent years, several studies of the impact of an intrinsic heavy quark component on the proton wave function in the $\gamma+Q$ and $Z+Q$ cross sections were performed [24-27], with particular emphasis on the case $Q=c$, since the probability of an intrinsic bottom is expected to be suppressed by a factor $\left(m_{c}^{2} / m_{b}^{2}\right)$ [11]. Our goal in this paper is to complement these previous studies by the analysis of the phenomenological implications of the intrinsic charm in the $Z$ boson production and related processes at the LHC energies. In particular, we will analyze the impact of the intrinsic charm in the rapidity and transverse momentum dependencies of the $Z, Z+$ jet, $Z+c$ and $Z+c+$ jet cross sections at $\sqrt{s}=7 \mathrm{TeV}$. The cross sections will be computed considering the next-to-leading order corrections using the partonlevel MC generator MCFM-version 6.8 [28] and using as input the different models present in the CT14 parton distributions [5]. It is important to emphasize that the MCFM does not take into account the mass corrections on the incoming heavy quark lines, which makes our calculations unreliable close to threshold. Such subjects have been discussed in the literature in the last years and advances on the treatment of the intrinsic heavy quarks in the perturbative computation of hard processes in the full kinematical range were recently presented in Ref. [29].

We will start our analysis discussing the different models for the intrinsic charm (IC) present in the CT14 parametrization [5]. As in Ref. [4], the recent analysis performed in [5] considered the intrinsic charm as an ingredient in the global fit of DIS and hadronic colliders data and determined the shape and normalization of the IC distribution in the same way as they do for other parton species. In fact one finds several IC distributions which are compatible with the world data. In addition to the already mentioned BHPS model, the CTEQ-TEA group has tested another model of intrinsic charm, called sea-like IC. It consists basically in assuming that at a very low resolution (before the DGLAP evolution) there is already some charm in the nucleon, which has a typical sea quark momentum distribution $(\simeq 1 / \sqrt{x})$ with normalization to be fixed by fitting data. In particular, they pro- 

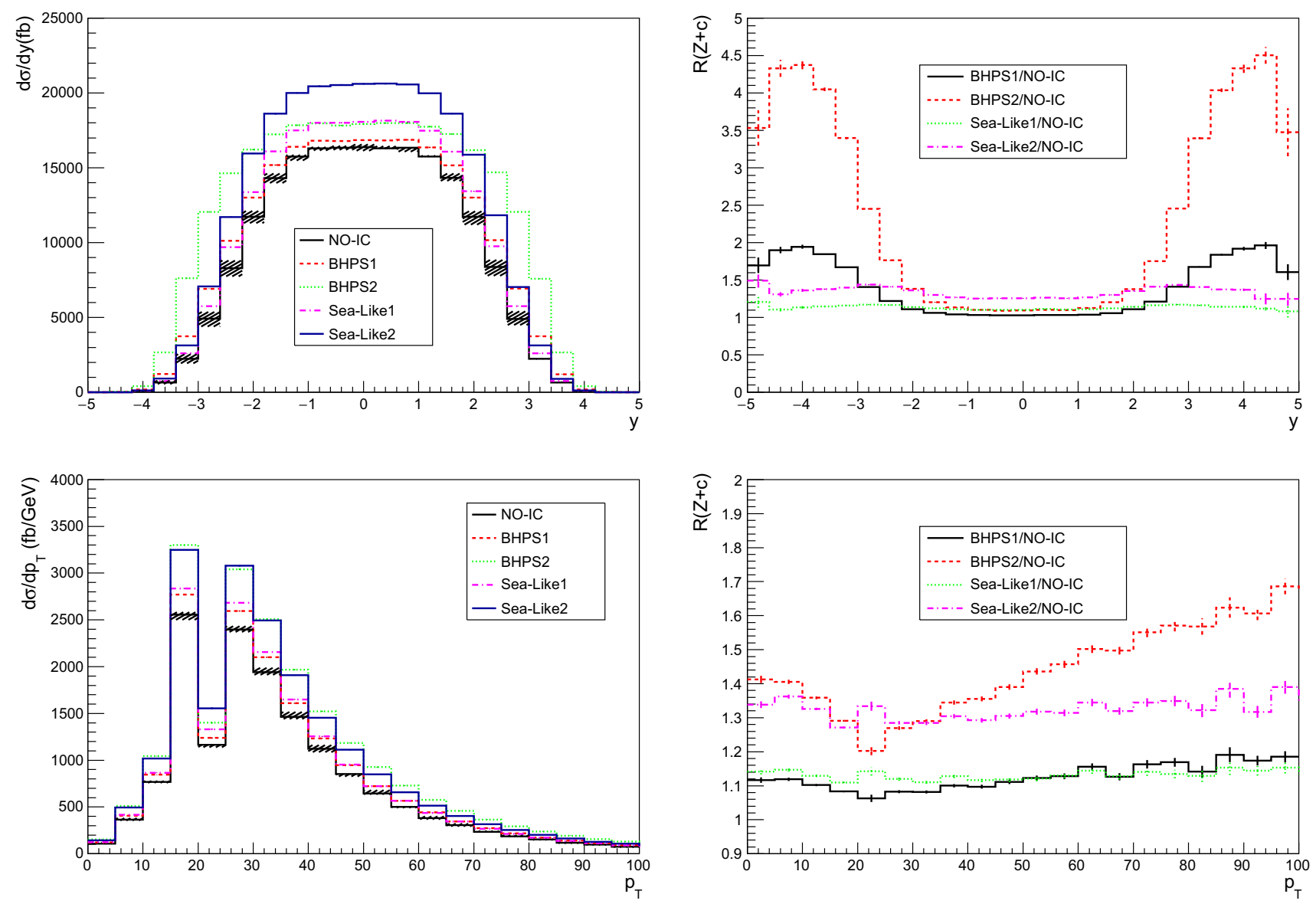

Fig. 4 Left panels Predictions for the rapidity and transverse momentum distributions for the $Z+c$ production in $p p$ collisions at $\sqrt{s}=7$ TeV. Right panels Rapidity and transverse momentum dependencies of the ratio between the IC and central NO-IC predictions

vided four different sets of PDFs which differ in the model for the IC and in the mean momentum fraction of the IC $\mathrm{PDF},\langle x\rangle_{I C}$, and which are compatible with the current world data. These four sets will be used as input in our calculations and will be denoted by BHPS1 $\left(\langle x\rangle_{I C}=0.6 \%\right)$, BHPS2 $\left(\langle x\rangle_{I C}=2.0 \%\right)$, Sea-like $1\left(\langle x\rangle_{I C}=0.6 \%\right)$, and Sea-like $2\left(\langle x\rangle_{I C}=1.5 \%\right)$. The different parametrizations are presented in Fig. 1 for two different values of the hard scale $Q^{2}$. For comparison we also present the NO-IC distribution, where the charm content of the nucleon sea comes from the DGLAP evolution (exclusive charm component). In this case we present the uncertainty band in this distribution obtained by the CTEQ-TEA group. For $Q^{2}=2.25 \mathrm{GeV}^{2}$ (left panel) the intrinsic charm distribution can be a factor 10 (Sea-like 1) to 20 (BHPS2) larger than the NO-IC distribution and the peaks of the IC distributions occurs in the large $x(\geq 0.1)$ region. For $Q^{2}=m_{Z}^{2}$ (right panel) the peaks decreases in magnitude and also shifts to smaller values of $x$. However, this peculiar behavior still is present, which gives us hope to observe IC experimentally in the charm-initiated processes.

In what follows we present our results for the rapidity and transverse momentum distributions for the $Z, Z+$ jet,
$Z+c$, and $Z+c+$ jet cross sections considering $p p$ collisions at $\sqrt{s}=7 \mathrm{TeV}$ and the different models for the intrinsic charm present in Ref. [5]. Moreover, for comparison we also will present the predictions obtained using as input only the extrinsic component of the charm distribution, denoted NOIC in the figures, taking into account the uncertainties present in the parton distributions derived by the CTEQ-TEA group. Consequently, our NO-IC predictions will be represented in the figures by a band of possible values for the cross sections. Finally, in order to estimate the contribution of the intrinsic charm for the process considered, we will also present the ratio between the IC and the central NO-IC predictions for the cross sections. The analysis of the rapidity and transverse momentum distributions of this ratio allows one to determine the kinematical range where the impact of the presence of an intrinsic component in the proton wave function is larger for the cross section. In Fig. 2 we present our predictions for the $Z$ production, which at leading order is proportional to $c\left(x_{1}\right) \bar{c}\left(x_{2}\right)+\bar{c}\left(x_{1}\right) c\left(x_{2}\right)$. We see that the effect of the intrinsic charm in the rapidity and transverse distribution distributions is small, as can be observed by the analysis of the ratio between IC and NO-IC predictions. Moreover, the uncer- 

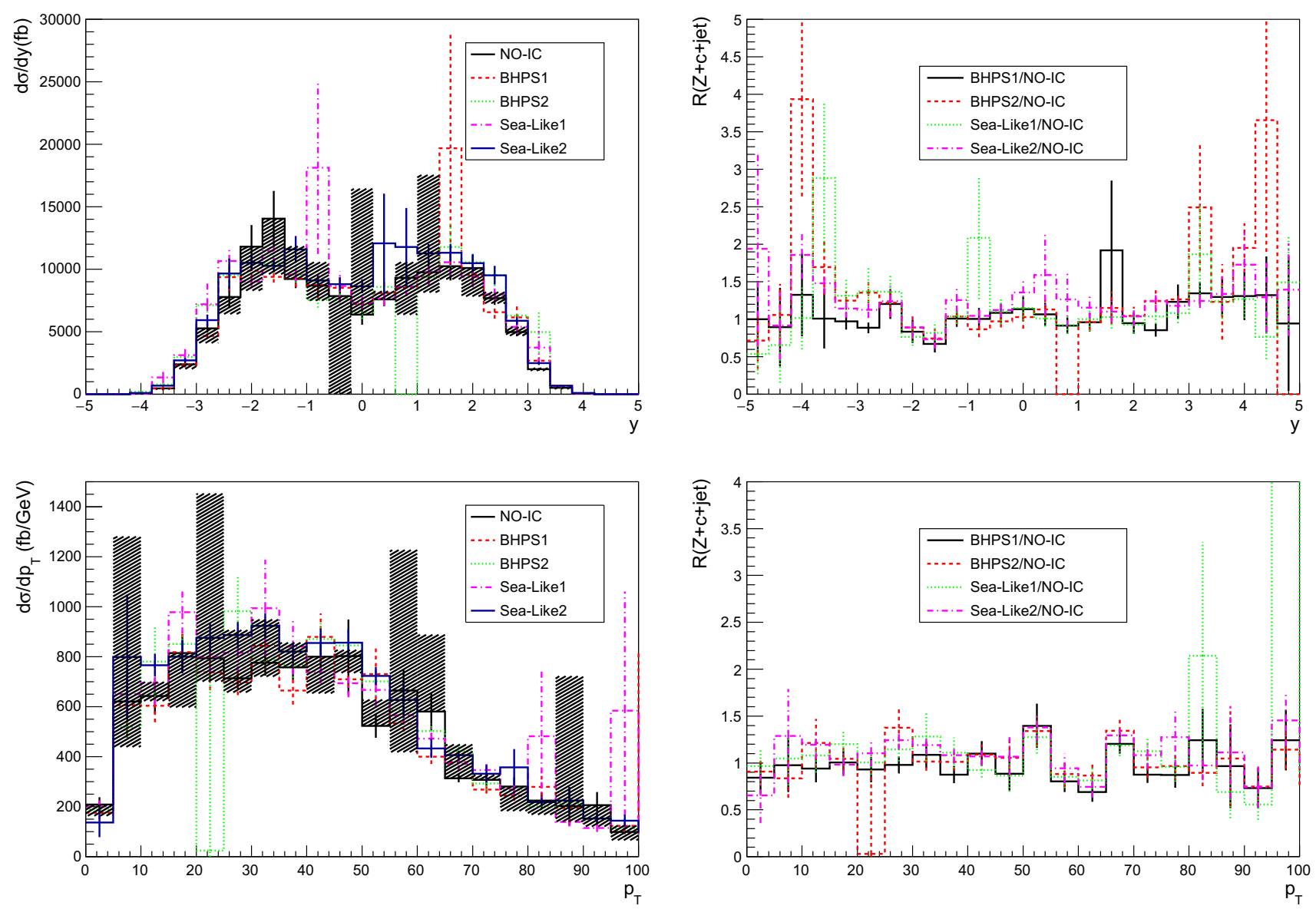

Fig. 5 Left panels Predictions for the rapidity and transverse momentum distributions for the $Z+c+$ jet production in $p p$ collisions at $\sqrt{s}=7$ $\mathrm{TeV}$. Right panels Rapidity and transverse momentum dependencies of the ratio between the IC and central NO-IC predictions

tainty band in the NO-IC predictions is small. These results are expected, since the cross section is dominated by the contribution of light quarks and the uncertainty in its PDFs at large hard scales is small. A similar conclusion is derived from the analysis of our predictions for the $Z+$ jet production presented in Fig. 3. However, in this case the uncertainty band is larger due to the contribution of the gluon initiated subprocesses. The small impact of the intrinsic charm in the $Z$ and $Z+$ jet production is an important aspect, which will be explored in the analysis of the ratio between the cross sections of different processes to be discussed below. In Fig. 4 we present our predictions for the $Z+c$ cross section [30], which for leading order is proportional to the partonic subprocess $g+c \rightarrow c+Z$ and consequently directly dependent on the charm content of the proton. We see that the uncertainty band in the NO-IC predictions is in general small, with the IC predictions being above the uncertainty. In agreement with previous studies [27], we see that the behavior of the transverse momentum distribution at large $p_{T}$ is modified by the presence of an intrinsic component in the charm distribution. In particular, the BHPS2 model implies an enhancement of a factor 1.7 at $p_{T}=100 \mathrm{GeV}$. In the case of the rapidity distribution, the intrinsic charm implies a large enhancement at forward rapidities, with the BHPS1 and BHPS2 predictions implying an increase by a factor of 2 and 4.5 for $y=4$. It is important to emphasize that such enhancements can be probed by the analysis of this process in the LHCb detector. Finally, in Fig. 5 we present our predictions for the $Z+c+$ jet cross section $[31,32]$. In this case the predictions are not strongly affected by an intrinsic charm component, which is associated to the fact that the diagrams where the charm is not present in the initial state contribute significantly for this process. Moreover, we see that this process is strongly affected by the uncertainty present in the NO-IC PDFs.

The previous results indicate that the ideal process to probe the existence of an intrinsic charm is the $Z+c$ production at large transverse momentum and forward rapidities, which is the kinematical range where the large- $x$ behavior of the charm PDF of the proton is probed. However, as the $Z+c$ cross section is sensitive to experimental and theoretical uncertainties, it is interesting to analyze other associated observables where these uncertainties are reduced. 
(a)

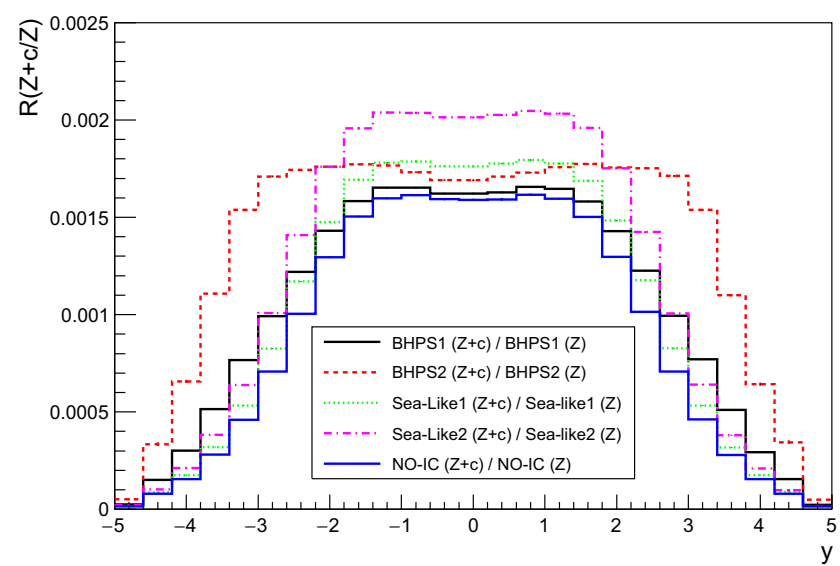

(b)

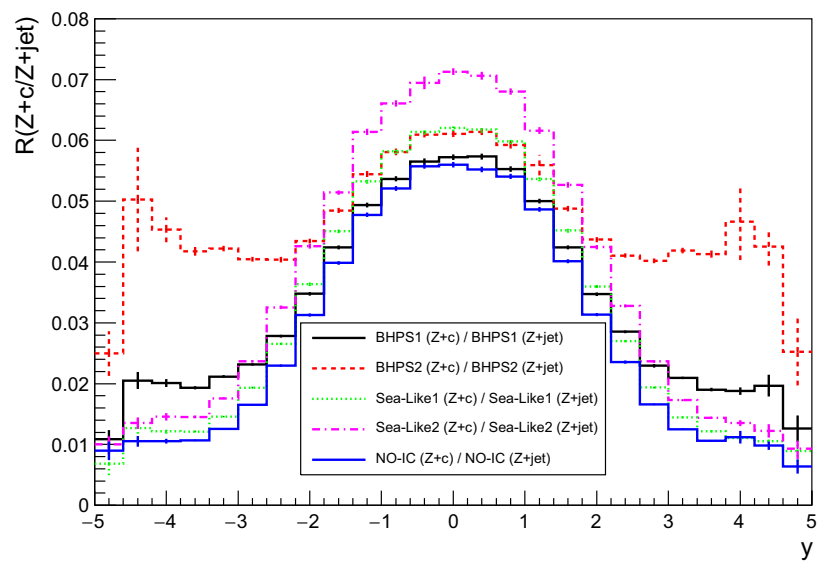

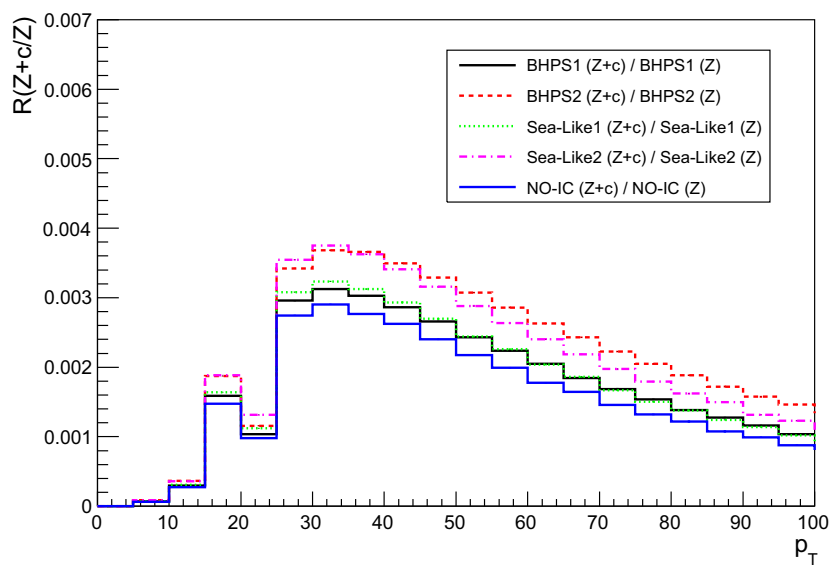

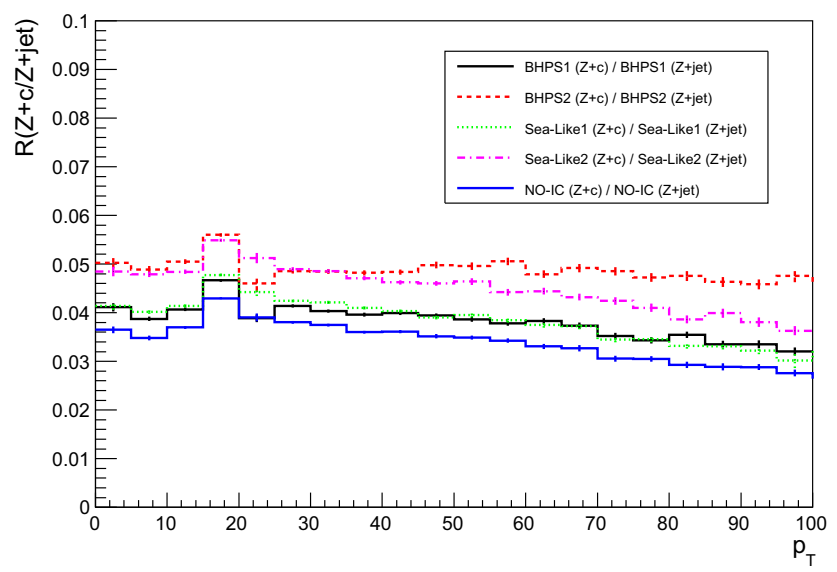

Fig. 6 Rapidity and transverse momentum dependencies of the ratios a $R(Z+c / Z) \equiv \sigma(Z+c) / \sigma(Z)($ upper panels $)$ and $\mathbf{b} R(Z+c / Z) \equiv$ $\sigma(Z+c) / \sigma(Z+$ jet $)$ (lower panels)

Here we propose the analysis of the rapidity and transverse momentum dependencies of the ratios $R(Z+c / Z) \equiv \sigma(Z+$ $c) / \sigma(Z)$ and $R(Z+c / Z+$ jet $) \equiv \sigma(Z+c) / \sigma(Z+$ jet $)$. An advantage of the analysis of these ratios is that PDFs uncertainties are strongly reduced, since numerator and denominator should be estimated using the same PDF set in order to be theoretically consistent. As demonstrated before, the $Z$ and $Z+$ jet cross sections are almost independent of the intrinsic charm, which implies that these ratios are directly dependent on the effects present in the $Z+c$ process. In Fig. 6 we present our predictions for these ratios, where we have estimated the different cross sections considering a common input for the PDFs. The baseline for comparison is the prediction obtained using the NO-IC PDFs as input in the calculations. In the case of the ratio $R(Z+c / Z)$, we see that the Sea-like models for the intrinsic charm implies that the ratio is enhanced by $10-20 \%$ at central rapidities, while the BHPS one implies an enhancement between 50 and $300 \%$ for $y=3$. Moreover, we see that the intrinsic charm implies an enhancement in the $p_{T}$ distribution of the ratio. Finally, for the ratio $R(Z+c / Z+$ jet $)$ we obtain a larger impact of the intrinsic charm, with analysis of this ratio at central (forward) rapidities being an important probe of the Sea-like (BHPS) models.

Let us summarize our main conclusions. Although the direct measurements of heavy flavors in DIS and hadronic colliders are consistent with a perturbative origin, these experiments are not sensitive to heavy quarks at large $x$. Therefore, it is fundamental to study other observables which may be used to determine the presence (or not) of an intrinsic heavy quark component in the hadron wave function. In recent years, a series of studies have discussed in detail the probe of this intrinsic component, with particular emphasis in processes that are strongly sensitive to the charm in the initial state. Our goal in this paper was to contribute for this theoretical effort by the analysis of $Z$-boson production and related processes in $p p$ collisions at the LHC. In particular, we have discussed the $Z, Z+$ jet, $Z+c$ and $Z+c+$ jet cross sections considering different models for the intrinsic charm. Our results indicated that differently from the other 
processes, the $Z+c$ cross section is strongly affected by the presence of the intrinsic charm. Moreover, we proposed the analysis of the ratios $R(Z+c / Z)$ and $R(Z+c / Z+$ jet $)$, and we demonstrated that these observables can be used as a probe of the intrinsic charm. Although we have presented results only for $\sqrt{s}=7 \mathrm{TeV}$, similar effects are expected for larger energies. Finally, our results indicated that the analysis of these processes at large transverse momentum and forward rapidities is fundamental to test the intrinsic charm hypothesis, which makes the LHCb experiment the ideal laboratory. This conclusion motivates a dedicated analysis considering the experimental characteristics of the LHCb detector, which we postpone for a future publication.

Acknowledgments We would like to thanks to M. S. Rangel for the helpful discussions in the earlier stages of this project. G. Bailas thanks G. G. da Silveira for the support in the numerical implementation of this study. This work was partially financed by the Brazilian funding agencies CNPq, CAPES and FAPERGS.

Open Access This article is distributed under the terms of the Creative Commons Attribution 4.0 International License (http://creativecomm ons.org/licenses/by/4.0/), which permits unrestricted use, distribution, and reproduction in any medium, provided you give appropriate credit to the original author(s) and the source, provide a link to the Creative Commons license, and indicate if changes were made. Funded by SCOAP ${ }^{3}$.

\section{References}

1. P. Newman, M. Wing, Rev. Mod. Phys. 86(3), 1037 (2014)

2. O. Behnke, A. Geiser, M. Lisovyi, Prog. Part. Nucl. Phys. 84, 1 (2015)

3. W.K. Tung et al., JHEP 0702, 053 (2007)

4. J. Pumplin, H.L. Lai, W.K. Tung, Phys. Rev. D 75, 054029 (2007)

5. S. Dulat, T.J. Hou, J. Gao, J. Huston, J. Pumplin, C. Schmidt, D. Stump, C.-P. Yuan, Phys. Rev. D 89(7), 073004 (2014)

6. P. Jimenez-Delgado, T.J. Hobbs, J.T. Londergan, W. Melnitchouk, Phys. Rev. Lett. 114(8), 082002 (2015)

7. F. Lyonnet, A. Kusina, T. Jeo, K. Kovark, F. Olness, I. Schienbein, J.Y. Yu, JHEP 1507, 141 (2015)
8. J. Pumplin, Phys. Rev. D 73, 114015 (2006)

9. W.C. Chang, J.C. Peng, Prog. Part. Nucl. Phys. 79, 95 (2014)

10. S.J. Brodsky, A. Kusina, F. Lyonnet, I. Schienbein, H. Spiesberger, R. Vogt, Adv. High Energy Phys. 2015, 231547 (2015)

11. S.J. Brodsky, P. Hoyer, C. Peterson, N. Sakai, Phys. Lett. B 93, 451 (1980)

12. S. Paiva, M. Nielsen, F.S. Navarra, F.O. Duraes, L.L. Barz, Mod. Phys. Lett. A 13, 2715 (1998)

13. F.S. Navarra, M. Nielsen, C.A.A. Nunes, M. Teixeira, Phys. Rev. D 54, 842 (1996)

14. F. Carvalho, F.O. Duraes, F.S. Navarra, M. Nielsen, Phys. Rev. Lett. 86, 5434 (2001)

15. T.J. Hobbs, J.T. Londergan, W. Melnitchouk, Phys. Rev. D 89(7), 074008 (2014)

16. S.J. Brodsky, S. Gardner, arXiv:1504.00969 [hep-ph]

17. G. Ingelman, M. Thunman, Z. Phys. C 73, 505 (1997)

18. B.A. Kniehl, G. Kramer, I. Schienbein, H. Spiesberger, Phys. Rev. D 79, 094009 (2009)

19. V.P. Goncalves, F.S. Navarra, T. Ullrich, Nucl. Phys. A 842, 59 (2010)

20. S.J. Brodsky, B. Kopeliovich, I. Schmidt, J. Soffer, Phys. Rev. D 73, 113005 (2006)

21. R. Vogt, S.J. Brodsky, Nucl. Phys. B 438, 261 (1995)

22. R. Vogt, S.J. Brodsky, Nucl. Phys. B, 478, 311 (1996)

23. V.D. Barger, F. Halzen, W.Y. Keung, Phys. Rev. D 25, 112 (1982)

24. T.P. Stavreva, J.F. Owens, Phys. Rev. D 79, 054017 (2009)

25. V.A. Bednyakov, M.A. Demichev, G.I. Lykasov, T. Stavreva, M. Stockton, Phys. Lett. B 728, 602 (2014)

26. S. Rostami, A. Khorramian, A. Aleedaneshvar, M. Goharipour, arXiv:1510.08421 [hep-ph]

27. P.H. Beauchemin, V.A. Bednyakov, G.I. Lykasov, Y.Y. Stepanenko, Phys. Rev. D 92(3), 034014 (2015)

28. J.M. Campbell, R.K. Ellis, Phys. Rev. D 65, 113007 (2002). http:// mcfm.fnal.gov/

29. R.D. Ball, V. Bertone, M. Bonvini, S. Forte, P. Groth Merrild, J. Rojo, L. Rottoli, Phys. Lett. B 754, 49 (2016)

30. J.M. Campbell, R.K. Ellis, F. Maltoni, S. Willenbrock, Phys. Rev. D 69, 074021 (2004)

31. J.M. Campbell, R.K. Ellis, F. Maltoni, S. Willenbrock, Phys. Rev. D 73, 054007 (2006)

32. J.M. Campbell, R.K. Ellis, F. Maltoni, S. Willenbrock, Phys. Rev. D 77, 019903 (2008) 